\title{
Research on Stress-Strength Model under Random Repeated Cycles
}

\author{
T.Sumathi Uma Maheswari, M.Tirumala Devi
}

\begin{abstract}
The system leads to fail when the impact of repeated stresses. In some situations there is uncertainty about the stress and the strength random variables at any instant of time and also about the behaviour of the random variables with respect to time and/or cycles. The repeated cycles may occur in known or unknown timings. The terms random fixed and random independent are used to describe these uncertainties. The survival function as the probability of survival of a system beyond any given time has been derived for the impact of repeated stresses and when the number of cycles occur in poisson distribution and geometric distribution when stress and strength follow random independent and also random fixed which follow weibull distribution with different parameters.
\end{abstract}

\section{INTRODUCTION}

Stress is used to indicate any agency that tends to induce failure while strength indicates any agency restricting failure.

Successive stresses are generally independent. Strengths will vary random and will be independent from cycle to cycle only. It is being affected by the environmental factors. Strength depends on the number of load applications, their magnitudes and time durations. If the strength varies only with time, the effect is called aging. When aging occurs the parameters of the variable change with respect to time. If the strength is a function of the number of load applications the effect is called cyclic damage. If the value of the strength depends on the number of load occurrences as well as their magnitude the effect is called cumulative damage. The strength may also depend on the sequence of loading, cumulative damage adds considerable complexity in the models. In addition we must consider the stress applications, frequency factor or the usage rate is done by converting $R_{n}$ to $R(t)$ under the Poisson assumption. The failure of a system under repeated stresses has been investigated with respect to the fatigue behaviour of the nature[1]. S N N Pandit et al [2] derived survival function under strength attenuation in cascade reliability. AC N Raghavachar et al [3] studied the survival function under stress attenuation in cascade reliability. Serkan Eryilmaz[4] discussed the stress strength reliability with a time dependent strength. K C Siju and M Kumar[5] discussed the reliability analysis of time dependent stress strength model with random cycle times. K C Siju and M Kumar[6] computed the reliability for a dynamic stress strength model with random cycle times.

\section{MATHEMATICAL MODEL \& RESULTS}

Survival function of $i^{t h}$ cycle of the component $=\mathcal{F}(i)$

$$
=\operatorname{Prob}\left(X_{i}>Y_{i}\right)
$$

Where $X_{i}$ is the strength random variability of the $i^{\text {th }}$ cycle and $Y_{i}$ is the stress of $i^{\text {th }}$ cycle of the component.

Let the probability of the occurrence of $i$ no. of cycles in the time interval $[0, t]$

$$
=\pi_{i}(t)
$$

Then the survival function at time $t$ is[1]

$$
\mathcal{F}(t)=\sum_{i=0}^{\infty} \pi_{i}(t) \mathcal{F}(i)
$$

If the number of cycles follows poisson distribution then $\pi_{i}(t)=\frac{e^{-a t}(a t)^{i}}{i !}, i=0,1, \ldots$

If the number of cycles follows geometric distribution then

$\pi_{i}(t)=p q^{i}, i=$

$0,1, \ldots$, where $p$ : probability of success, $q=1-p$

Case (i): Random fixed stress and random independent strength

In this case, the stress $Y$ is random variable with known probability density function $g(y)$ and stress for $i^{\text {th }}$ cycle be $Y_{i}=\frac{Y}{\mu_{i}^{*}}$ and successive random strengths $X_{1}, X_{2}, \ldots, X_{i}$ are random independent and i.i.d with probability density function $f(x)$ then

survival function of $i^{\text {th }}$ cycle $=\mathcal{F}(i)=P\left[E_{1}, E_{2}, \ldots, E_{i}\right]$ The event

$E_{i}=$ the compnent survival at $i^{\text {th }}$ cycle i.e., $E_{i} \sim X_{i}>Y$.

Then the survival function of the component for $i^{\text {th }}$ cycle

$$
\begin{gathered}
\mathcal{F}(i)=P\left[\left(X_{1}>\frac{Y}{\mu_{1}^{*}}\right),\left(X_{2}>\frac{Y}{\mu_{2}^{*}}\right), \ldots,\left(X_{i}>\frac{Y}{\mu_{i}^{*}}\right)\right] \\
=P\left[\bigcap_{i=1}^{n}\left(X_{i}>\frac{Y}{\mu_{i}^{*}}\right)\right]
\end{gathered}
$$

Since all $X_{i}$ 's are independent and identical then

$$
\begin{aligned}
\mathcal{F}(i) & =\int_{-\infty}^{\infty}\left[\int_{x=\frac{Y}{\mu_{i}^{*}}}^{\infty} f(x) d x\right]^{i} g(y) d y \\
& =\int_{0}^{\infty}\left[1-F\left(\frac{Y}{\mu_{i}^{*}}\right)\right]^{i} g(y) d y
\end{aligned}
$$

Then the survival function at time $t$ is 


$$
\mathcal{F}(t)=\sum_{i=0}^{\infty} \pi_{i}(t) \mathcal{F}(i)
$$

If number of cycles follows poisson distribution then

$$
\mathcal{F}(t)=\sum_{i=0}^{\infty} \frac{e^{-a t}(a t)^{i}}{i !} \int_{0}^{\infty}\left[1-F\left(\frac{Y}{\mu_{i}^{*}}\right)\right]^{i} g(y) d y
$$

If number of cycles follows geometric distribution then

$$
\mathcal{F}(t)=\sum_{i=0}^{\infty} p q^{i} \int_{0}^{\infty}\left[1-F\left(\frac{Y}{\mu_{i}^{*}}\right)\right]^{i} g(y) d y
$$

If strength $X$ and stress $Y$ follow weibull distribution with probability density functions $f(x)$ and $g(y)$ respectively then

$$
\begin{gathered}
f(x)=k x^{m} e^{-\frac{k x^{m+1}}{m+1}}, x>0, \quad k, m>0 \\
g(y)=l y^{m} e^{-\frac{l y^{m+1}}{m+1}}, y>0, \quad l, m>0 \\
\mathcal{F}(x)=\int_{0}^{x} f(x) d x \\
=\int_{0}^{x} k x^{m} e^{-\frac{k x^{m+1}}{m+1}} d x \\
=1-\left[e^{-\frac{k x^{m+1}}{m+1}}\right]
\end{gathered}
$$

When $\mu_{i}^{*}=1$ then

$$
\begin{aligned}
\mathcal{F}(i) & =\int_{0}^{\infty} l y^{m} \exp \left(-\frac{(k i+l) y^{m+1}}{m+1}\right) d y \\
& =\frac{l}{k i+l}
\end{aligned}
$$

If the number of cycles follows poisson distribution then

$$
\begin{aligned}
\mathcal{F}(t) & =e^{-a t} \sum_{i=0}^{\infty} \frac{(a t)^{i}}{i !} \frac{l}{(i k+l)} \\
& =e^{-a t}\left[1+a t \frac{l}{(k+l)}+\frac{(a t)^{2}}{2 !} \frac{l}{(2 k+l)}+\cdots\right]
\end{aligned}
$$

In special case, $l=k$ then $\mathcal{F}(t)=\frac{1}{a t}$ where a is mean number of cycles

If the number of cycles follows geometric distribution then

$$
\mathcal{F}(t)=\sum_{i=0}^{\infty} p q^{i} \frac{l}{(i k+l)}
$$

In special case, $l=k$ then

$$
\mathcal{F}(t)=p\left[1+\frac{q}{2}+\frac{q^{2}}{3}+\cdots\right]=p \sum_{i=0}^{n} \frac{q^{i}}{i+1}
$$

Case (ii): Random independent stress and random independent strength

Let $f(x) \& g(y)$ represent the probability density functions of strength $X$ and stress $Y$ respectively then survival probability for one cycle is

$$
\mathcal{F}=\int_{0}^{\infty} f(x)\left[\int_{y=0}^{x} g(y) d y\right] d x
$$

Since the random variables be independent and identical for each cycle. Then survival function for ith cycle

$$
\mathcal{F}(i)=\prod_{i=1}^{i} \mathcal{F}=\mathcal{F}^{i}, i=1,2, \ldots
$$

when the number of cycles follows poisson distribution then

$$
\begin{aligned}
\mathcal{F}(t) & =\sum_{i=0}^{\infty} \pi_{i}(t) \mathcal{F}(i) \\
& =\sum_{i=0}^{\infty} \frac{e^{-a t}(a t)^{i}}{i !} \mathcal{F}^{i} \\
& =e^{-a t} \sum_{i=0}^{\infty} \frac{(a t \mathcal{F})^{i}}{i !} \\
& =e^{-a t} e^{a t \mathcal{F}} \\
& =e^{-a t(1-\mathcal{F})}
\end{aligned}
$$

and

$$
\begin{aligned}
\mathcal{F} & =\int_{0}^{\infty} k x^{m} e^{-\frac{k x^{m+1}}{m+1}} \int_{0}^{x}\left[l y^{m} e^{-\frac{l y^{m+1}}{m+1}} d y\right] d x \\
& =\frac{l}{l+k}
\end{aligned}
$$

Therefore survival function at time $t$ is

$$
\mathcal{F}(t)=e^{-a t}\left(\frac{k}{k+l}\right)
$$

In a special case, if $l=k$ then $\mathcal{F}(t)=e^{-\left(\frac{1}{2}\right) a t}$

If the number of cycles follows geometric distribution then

$$
\begin{aligned}
\mathcal{F}(t) & =\sum_{i=0}^{\infty} p q^{i} \mathcal{F}^{i} \\
& =p \sum_{i=0}^{\infty}\left[\frac{q l}{l+k}\right]^{i}
\end{aligned}
$$

If $l=k$ then

$$
\begin{aligned}
\mathcal{F}(t) & =p \sum_{i=0}^{\infty}\left[\frac{q}{2}\right]^{i} \\
& =\frac{2 p}{2-q}
\end{aligned}
$$

Case (iii): Random fixed stress and random fixed strength

Let $x_{0} \& y_{0}$ be random fixed strength and stress and probability density functions are $f_{0}\left(x_{0}\right) \& g_{0}\left(y_{0}\right)$ respectively. Then survival function of $i^{\text {th }}$ cycle of a component is

$$
\mathcal{F}(i)=\int_{0}^{\infty} f_{0}\left(x_{0}\right)\left[\int_{0}^{x_{0}} g_{0}\left(y_{0}\right) d y_{0}\right] d x_{0}
$$

If $X$ and $Y$ follow Weibull distribution then

$$
\begin{aligned}
\mathcal{F}(i) & =\int_{0}^{\infty} k x_{0}^{m} e^{-\frac{k x_{0} m+1}{m+1}}\left[\int_{0}^{x_{0}}\left[l y_{0}^{m} e^{-\frac{l y_{0} m+1}{m+1}} d y_{0}\right]\right] d x_{0} \\
& =\frac{l}{l+k}
\end{aligned}
$$

Hence

$$
\mathcal{F}(t)=\sum_{i=0}^{\infty} \pi_{i}(t) \mathcal{F}(i)
$$

Published By: 


$$
\begin{aligned}
& =\pi_{0}(t) \mathcal{F}(0) \\
& +\sum_{i=1}^{\infty} \pi_{i}(t) \mathcal{F}(i)
\end{aligned}
$$

Then survival function at initial time $=\mathcal{F}(0)=1$ and $\mathcal{F}(i)=\mathcal{F}$

If the number of cycles follows poisson distribution then

$$
\begin{gathered}
\mathcal{F}(t)=e^{-a t}+\mathcal{F}\left(1-e^{-a t}\right) \\
\mathcal{F}(t)=e^{-a t}+\frac{l}{l+k}\left(1-e^{-a t}\right) \\
=e^{-a t}\left(\frac{k}{k+l}\right)+\frac{l}{l+k}
\end{gathered}
$$

If $l=k$ then $\mathcal{F}(t)=\frac{1}{2}\left(1+e^{-a t}\right)$

If the number of cycles follows geometric distribution then from $(A)$

$$
\begin{gathered}
\mathcal{F}(t)=p+\mathcal{F}(1-p) \\
=p+\frac{l}{l+k}(1-p)
\end{gathered}
$$

If $l=k$ then $\mathcal{F}(t)=\frac{p+1}{2}$

The strength and stresses acting on a component on various cycles are independent and identically distributed as weibull random variables with parameters $\mathrm{k}, \mathrm{m}$ and $1, \mathrm{~m}$. Finding the survival probabilities of the system as a function of $\mathrm{t}$ for number of cycles follows poisson and geometric distribution for various parameters mean number of cycles(a), time(t), stress parameter(l),strength parameter(k), probability of success of the cycle(p).

\section{NUMERICAL COMPUTATIONS OF SURVIVAL FUNCTION FOR VARIOUS PARAMETERS}

Case (i): Random fixed stress and random independent strength

a) Number of cycles follows poisson distribution $t=5, l=0.5, k=0.1$

\begin{tabular}{|c|c|c|c|c|c|c|c|c|c|}
\hline$a$ & 0.1 & 0.2 & 0.3 & 0.4 & 0.5 & 0.6 & 0.7 & 0.8 & 0.9 \\
\hline $\mathcal{F}(t)$ & 0.921304 & 0.844152 & 0.759788 & 0.66701 & 0.569923 & 0.474311 & 0.355253 & 0.305133 & 0.235558 \\
\hline
\end{tabular}

$a=0.1, l=0.5, k=0.1$

\begin{tabular}{|c|c|c|c|c|c|c|c|c|c|}
\hline$t$ & 5 & 6 & 7 & 8 & 9 & 10 & 11 & 12 & 13 \\
\hline$F(t)$ & 0.921304 & 0.966127 & 0.899965 & 0.87355 & 0.859956 & 0.844152 & 0.829020 & 0.811503 & 0.7946 \\
\hline
\end{tabular}

$a=0.1, t=5, k=0.1$

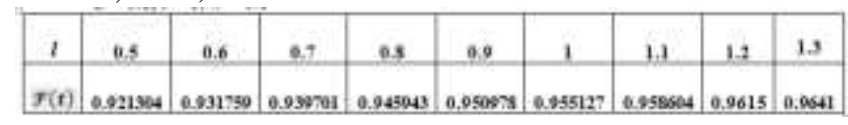

\begin{tabular}{|c|c|c|c|c|c|c|c|c|c|}
\hline$I$ & 1 & 2 & 3 & 4 & 5 & 6 & 7 & $s$ & 9 \\
\hline$F(t)$ & 0.23307 & 0.319113 & 0.396163 & 0.47200 & 0.54655 & 0.6211116 & 1.69844 & 0.76575 & 0.8423 \\
\hline
\end{tabular}
$a=0.1, t=5, l=0.5$

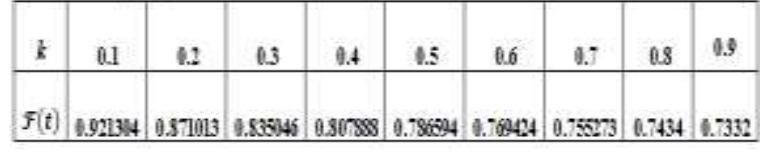

b) Number of cycles follows geometric distribution $l=0.5, k=0.2$

\begin{tabular}{|c|c|c|c|c|c|c|c|c|c|}
\hline$p$ & 0.1 & 0.2 & 0.3 & 0.4 & 0.5 & 0.6 & 0.7 & 0.3 & 0.9 \\
\hline$q$ & 0.9 & 0.8 & 0.7 & 0.6 & 0.5 & 0.4 & 0.3 & 0.2 & 0.1 \\
\hline $\mathcal{F}(t)$ & 0.21974 & 0.40419 & 0.556935 & 0.67949 & 0.77645 & 0.95062 & 0.9155 & 0.94455 & 0.9799 \\
\hline
\end{tabular}

$$
p=0.1, q=0.9, k=0.5
$$

$$
p=0.1, q=0.9, l=5
$$

\begin{tabular}{|c|c|c|c|c|c|c|c|c|c|}
\hline$t$ & 5 & 6 & 7 & 8 & 9 & 10 & II & 12 & 13 \\
\hline$F(t)$ & 0.91588 & 092329 & 0914112 & 0.915016 & 0.596011 & 0.8879\% & 0.578069 & 0.8655 & 0.8518 \\
\hline
\end{tabular}

\begin{tabular}{|c|c|c|c|c|c|c|c|c|c|}
\hline$k$ & 0.1 & 0.2 & 0.3 & 0.4 & 0.5 & 0.6 & 0.7 & 0.8 & 0.9 \\
\hline $\mathcal{F}(t)$ & 0.85916 & 0.74107 & 0.554 .66 & 1.59514 & 0.545051 & 0.565014 & 0.47653 & 0.45004 & 0.427 \\
\hline
\end{tabular}

Case (ii): Random independent stress and random independent strength

a) Number of cycles follows poisson distribution $t=5, l=0.01, k=0.5$

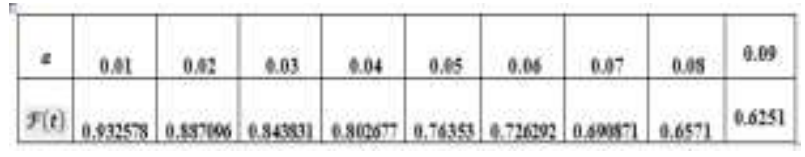

$a=0.01, l=0.01, k=0.5$

$a=0.01, t=5, k=0.5$

\begin{tabular}{|c|c|c|c|c|c|c|c|c|c|}
\hline$l$ & 0.01 & 0.02 & 0.03 & 0.04 & 0.05 & 0.06 & 0.07 & 0.08 & 0.69 \\
\hline $\mathcal{F}(t)$ & 0.93557 & 0.91464 & 0.597386 & 0.850768 & 0.86475 & 0.849312 & 0.834412 & 0.8200 & 0.9961 \\
\hline
\end{tabular}

$a=0.01, t=5, l=0.01$

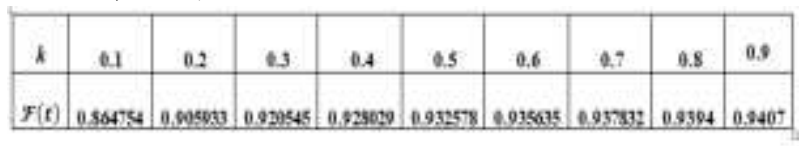

b) Number of cycles follows geometric distribution $l=0.2, k=0.5$

\begin{tabular}{|c|c|c|c|c|c|c|c|c|c|}
\hline$p$ & 0.1 & 0.2 & 0.3 & 0.4 & 0.5 & 0.6 & 0.7 & 0.3 & 0.9 \\
\hline$f$ & 0.9 & 0.8 & 0.7 & 0.6 & 0.5 & 0.4 & 0.3 & 0.2 & 0.1 \\
\hline$F(t)$ & 0.114723 & 0.225618 & 0.33956 & 0.445916 & 0.5555 & 0.453495 & 0.756733 & 0.84506 & 0.9310 \\
\hline
\end{tabular}
$p=0.1, q=0.9, k=5$

Published By: 


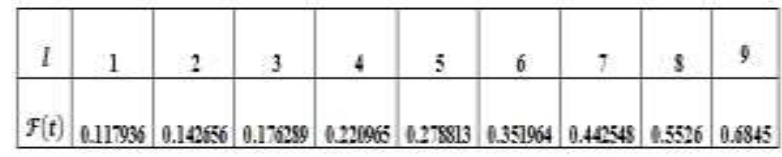

$p=0.1, q=0.9, l=0.5$

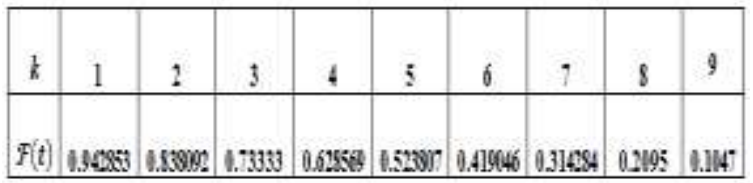

Case (iii): Random fixed stress and random fixed strength

a) Number of cycles follows poisson distribution $t=3, l=0.2, k=0.1$

\begin{tabular}{|c|c|c|c|c|c|c|c|c|c|}
\hline$\&$ & 0.1 & 0.2 & 0.3 & 0.4 & 0.5 & 0.6 & 0.7 & 0.5 & 0.9 \\
\hline $\mathcal{F}(t)$ & 0.912006 & 0.84964 & 0.80219 & 0.76766 & 0.741443 & 0.72176 & 0.7074 & 0.6969 & 0.6890 \\
\hline
\end{tabular}

$a=0.1, l=0.2, k=0.1$

\begin{tabular}{|c|c|c|c|c|c|c|c|c|c|}
\hline$t$ & 2 & 4 & 6 & 8 & 10 & 12 & 14 & 16 & 18 \\
\hline$F(t)$ & 0.995977 & 0.890107 & 0.849844 & 0.816449 & 0.789299 & 0.767065 & 0.748656 & 0.7339 & 0.7217 \\
\hline
\end{tabular}

$a=0.1, t=5, k=0.1$

\begin{tabular}{|c|c|c|c|c|c|c|c|c|c|}
\hline$l$ & 0.1 & $0 ?$ & 1.3 & 0.4 & 1.5 & 06 & 0.7 & 0.8 & 19 \\
\hline$F$ & 696 & 186834 & 6.901633 & 0921366 & 0.93442 & 094379 & 0.056816 & 09562 & 0.960 \\
\hline
\end{tabular}

$a=0.1, t=5, l=0.2$

\begin{tabular}{|c|c|c|c|c|c|c|c|c|c|}
\hline$k$ & 0.01 & 092 & 9.13 & 0.94 & 0.15 & 0.16 & 0.97 & 0.00 & 0.19 \\
\hline$F(t)$ & 0.9811263 & 0.96413 & 0.945678 & 0934422 & 1921166 & $0.5095 y$ & 0.899259 & 0.88758 & 0.5778 \\
\hline
\end{tabular}

b) Number of cycles follows geometric distribution $l=0.5, k=0.1$

\begin{tabular}{|c|c|c|c|c|c|c|c|c|c|}
\hline$p$ & 0.1 & 0.2 & 0.3 & 0.4 & 0.5 & 0.6 & 0.7 & 0.8 & 0.9 \\
\hline$q$ & 0.9 & 0.8 & 0.7 & 0.6 & 0.5 & 0.4 & 0.3 & 0.2 & 0.1 \\
\hline $\mathcal{f}(t)$ & 0.85 & 0.866607 & 0.893333 & 0.9 & 0.91666 & 0.93333 & 0.95 & 0.96665 & 0.9533 \\
\hline
\end{tabular}

$p=0.1, q=0.9, k=0.1$

\begin{tabular}{|c|c|c|c|c|c|c|c|c|c|}
\hline$l$ & 0.1 & 0.2 & 0.3 & 0.4 & 0.5 & 0.6 & 0.7 & 0.8 & 0.9 \\
\hline$F(t)$ & 0.55 & 0.7 & 0.775 & 0.82 & 0.85 & 0.57149 & 0.8875 & 0.9 & 0.91 \\
\hline
\end{tabular}

$p=0.1, q=0.9, l=0.1$

\begin{tabular}{|c|c|c|c|c|c|c|c|c|c|}
\hline$k$ & 0.1 & 0.2 & 0.3 & 0.4 & 0.5 & 0.5 & 0.7 & 0.5 & 0.9 \\
\hline$F(t)$ & 0.55 & 0.4 & 0.325 & 0.28 & 0.25 & 0.22551 & 0.215 & 0.2 & 0.19 \\
\hline
\end{tabular}

\section{CONCLUSION}

The survival function of the system for stress strength model of repeated stresses when random number of cycles if number of cycles follow poisson and geometric distribution have been derived. It is observed that when number of cycles increased reliability decreased. Strength parameter increases, Stress parameter decreases reliability is decreased.

\section{REFERENCES}

1. K C Kapur and L R Lamberson, 1977, Reliability in Engineering Design, John Wiley and Sons Inc New York.

2. S N N Pandit, A C N Raghavachar and B Kesava Rao, 1988, Survival function under strength attenuation in cascade reliability, IAPQR Transactions, vol 13, No.2.

3. A C N Raghavachar, B Kesava Rao and N Ch Pattabiramacharyulu, 1983, Survival function under stress attenuation in cascade reliability, OPSEARCH, vol 20, no 4, 190-207.

4. Serkan Erilmaz, 2013, On stress-strength reliability with a time-dependent strength, Journal of Quality and Reliability Engineering.

5. K C Siju and M Kumar, 2016, Reliability analysis of time dependent stress-strength model with random cycle times, Perspectives in Science, 8, 654-657.

6. K C Siju and M Kumar, 2017, Reliability computation of a dynamic stress-strength model with random cycle times, International Journal of Pure and Applied Mathematics, v01.117, no.12, 309-316. 